\title{
LA INTEGRACIÓN DE LA COMPETENCIA ESTRATÉGICA EN EL CURRÍCULO DE LENGUA EXTRANJERA
}

\author{
Susana Martín Leralta \\ Universität Bielefeld \\ susana.martin-leralta@uni-bielefeld.de
}

\begin{abstract}
Resumen
El desarrollo de la competencia estratégica en el aula, como medio para fomentar la autonomía del aprendiz, es uno de los actuales retos de la labor diaria docente, de acuerdo con las directrices de las instituciones y organismos reguladores de la enseñanza de lenguas extranjeras. Si bien la bibliografia sobre estrategias de aprendizaje y comunicación es abundante en cuanto a la naturaleza y posibles efectos ventajosos de las mismas, no responde a los requerimientos de la acción didáctica. Del mismo modo, las conclusiones de los estudios de intervención en el aula resultan difíciles de extrapolar a contextos de enseñanza diferentes a los del modelo de entrenamiento original, lo que implica la necesidad de adaptarlos a la situación concreta de aprendizaje formal en la que quiere ejercitarse el uso de las estrategias. En este sentido, se precisa de una secuencia de pautas que orienten al profesor en la inclusión del componente estratégico dentro del currículo de idiomas al servicio del resto de competencias lingüísticas, sin convertirlo en el eje vertebrador del mismo. El presente artículo avanza en esta dirección.

PALABRAS CLAVE: estrategias, competencia estratégica, entrenamiento, aprendizaje de lengua extranjera.
\end{abstract}

\begin{abstract}
According to the guidelines of institutions and organizations that regulate foreign language instruction, one of the challenges of today's teaching presents the development of strategic competence as a way to encourage the student's autonomy. However, in spite of a wide range of literature on the nature of learning and communication strategies and their possible advantages, these studies do not take didactic requirements into account. At the same time, conclusions of research on strategic training in class are difficult to transfer from the original teaching model to another learning context. This implies the necessity of adapting them to specific situations of formal learning in which these strategies are to be practiced. Thus, it is essential to establish a series of guidelines which serve as an orientation for the teacher in integrating the strategic component into the foreign language syllabus without neglecting other linguistic competences and transforming it into the main focus of the curriculum. This article aims to advance in such direction..
\end{abstract}

KEY WORDS: strategies, strategic competence, training, foreign language learning.

\section{Introducción}

Las demandas actuales a las que debe responder la enseñanza formal de lenguas modernas han colocado a la competencia estratégica en el punto de mira de investigadores y docentes. Los cambios sociales han modificado las concepciones tradicionales de aprendizaje lingüístico al originar nuevas necesidades y, en consecuencia, las bases teóricas que sustentan la actividad didáctica han sido replanteadas para dar cabida a otros requerimientos, entre ellos, el fomento de la autonomía del alumno de cara a promover el aprendizaje continuo o lifelong learning. 
De esta forma, el desarrollo de las estrategias de aprendizaje y comunicación en el aula de lenguas segunda y extranjera se ha convertido en uno de los objetivos del currículo de idiomas modernos' porque, aunque no sean un fin en sí mismas, ayudan a agilizar, mejorar y ampliar el resto de las competencias del aprendiz con vistas al desarrollo de su independencia.

Tomando en consideración las investigaciones realizadas desde los años ochenta, así como las recomendaciones de las instituciones y organismos reguladores de los sistemas educativos, nada habría que objetar a un programa de enseñanza de lenguas que incluyera el desarrollo explícito de la competencia estratégica; sin embargo, ésta queda relegada a un segundo plano en la realidad diaria del aula. La razón reside, principalmente, en la complejidad de la integración de la competencia estratégica en la práctica didáctica habitual (Grenfell y Harris, 1999; Harris y Gaspar, 2001; Macaro, 2001), puesto que no existe una secuencia de pautas de instrucción generalmente aceptada que el docente pueda aplicar directamente, sino que ha de adaptar a la situación de aprendizaje de sus alumnos alguno de los múltiples modelos de instrucción ofrecidos por la bibliografía.

Con objeto de favorecer la labor docente, el presente artículo revisa los conceptos de competencia estratégica y de estrategia, de manera que se posibilite su efectiva transmisión didáctica; señala las ventajas y riesgos de su inclusión en el currículo de LE, y presenta una serie de pautas encaminadas a facilitar la integración.

\section{La competencia estratégica y las estrategias del aprendiz}

Las investigaciones pioneras sobre competencia estratégica han sido revisadas y actualizadas para ofrecer un marco teórico óptimo para la acción didáctica. El concepto tradicional descriptivo (Canale y Swain, 1980; Canale, 1983, entre otros) se ha ampliado para explicar los mecanismos mediante los que operan las estrategias, así como para extender su función más allá de la mera resolución de problemas en la comunicación.

En este sentido, la competencia estratégica juega un papel central en la reformulada habilidad lingüistica comunicativa de Bachman (1990: 107-108):

Strategic competence is seen as the capacity that relates language competence, or knowledge of language, to the language user's knowledge structures and the features of the context in which communication takes place. Strategic competence performs assessment, planning, and execution functions in determining the most effective means of achieving a communicative goal.

La investigación de las estrategias en el campo de adquisición de lenguas segunda y extranjera se ha visto a lo largo de su desarrollo acompañada de una discusión, que no se puede dar por cerrada, en cuanto a la definición y delimitación del propio término estrategia, que ha impedido el establecimiento de una base teórica compartida que justifique los intentos de transmisión didáctica de las mismas (Ellis, 1997; Westhoff, 2001; Hill, 2004).

Para establecer una terminología que sirva a nuestros propósitos de aplicación didáctica, analizaremos el término estrategia desde las tres dimensiones que señala Wolff (1998) y que afectan a la instrucción formal en el aula: la psicológica, la didáctica y la técnico-cultural. 
Como concepto psicológico, las estrategias se consideran planes que se trazan para llevar a cabo operaciones complejas de resolución de problemas, en las cuales son de gran importancia la formación y la prueba de hipótesis. En tanto que operaciones mentales complejas, las estrategias controlan otras más simples (que podríamos denominar tácticas y técnicas, en este orden); respecto a la dimensión didáctica, por estrategias se entienden aquellos modos de actuación planeados que el aprendiz puede poner en marcha de manera más o menos consciente y cuyo fomento en el aula influye positivamente en su comportamiento, mientras que, en cuanto a la tercera dimensión, la técnico-cultural, las estrategias serían técnicas generales adquiridas durante la socialización para el manejo de la información como, por ejemplo, la lectura de mapas o la interpretación de gráficos y análisis estadísticos.

La coexistencia de estras tres dimensiones explica la diferente utilización de términos en la bibliografia, una situación que ha originado que, con frecuencia, se aplicara la etiqueta de estrategia de manera indiscriminada a operaciones cognitivas y recursos de distintos niveles de abstracción sin una base teórica que lo justificara ${ }^{2}$.

Del mismo modo, y para evitar la confusión entre los términos estrategias de aprendizaje y estrategias de comunicación, algunos investigadores se decantan por el término estrategias del aprendiz (Wenden, 1991; Töhnshoff, 1992; Cohen, 1998) para abarcar, así, todas las que puede poner en práctica el individuo, independientemente del contexto de adquisición de la lengua, de la finalidad de la estrategia o de si una tarea con fines comunicativos puede resultar en aprendizaje lingüístico. En el presente artículo adoptamos esta denominación.

De cara a delimitar el concepto de estrategia, resulta adecuado el procedimiento inductivo de Wenden (1987) a partir de sus rasgos definitorios. En este sentido, se exponen a continuación las particularidades que caracterizan a las estrategias tomando como criterio su relación con diferentes factores, a saber, respecto a su uso, al aprendizaje en general y el de la lengua en particular, al aprendiz, a otras variables que intervienen en el proceso de aprendizaje de una LE, como la tarea, el contexto y el profesor, y a la comunicación.

En cuanto a su uso, cabe afirmar que se trata de acciones concretas y específicas, no consistentes en orientaciones generales como puedan ser el carácter reflexivo del aprendiz o su disposición a asumir riesgos (Wenden, 1991; Oxford, 1990), por lo que se pueden individualizar. Sin embargo, cada una de ellas comprende un conjunto sistemático de medidas o elementos particulares que se relacionan entre sí con una finalidad común (Rampillon, 1985). Asimismo, destaca la doble naturaleza, activa y cognitiva, de las estrategias; es decir, aunque su ejecución implique la acción misma, también conlleva un movimiento de reflexión o la posibilidad de activarlo (Villanueva, 1997).

Por otra parte, el empleo de las estrategias debe contemplarse en relación con la problematicidad de la situación de uso lingüístico. Si Faerch y Kasper (1984b), Oxford (1990) y Wenden (1991) consideraban que las estrategias se ponían en marcha cuando el aprendiz o hablante no nativo encontraba un problema de aprendizaje o comunicación en LE, Bialystok (1990) acepta que se pueda producir en ausencia de problematicidad y movido, simplemente, por la intención de mejorar en la comunicación o de alcanzar un resultado óptimo con

2 Esta crítica la encontramos en Tönshoff (1992: 243) quien se refiere, entre otros, a Wenden (1987) y a Oxford (1990) para ejemplificar la falta de trasfondo teórico en las explicaciones del término. 
menos esfuerzo. El uso de la lengua en ausencia de problematicidad no sería menos estratégico que ante la aparición de una dificultad.

Finalmente, el uso de las estrategias se caracteriza por ser modificable, es decir, "flexible" (Oxford, 1990) o de "dimensión dinámica e interactiva" (Villanueva, 1997).

De acuerdo con el segundo de los criterios adoptados, la relación de las estrategias con el aprendizaje, debe señalarse que lo apoyan tanto directa como indirectamente e implican varios aspectos del mismo, a saber, el cognitivo (referido al conocimiento lingüístico), el metacognitivo (la capacidad del individuo para controlar y dirigir su propio aprendizaje de manera consciente) y el socioafectivo (relacionado con ítems motivacionales y creencias del aprendiz sobre sí mismo, la tarea de aprendizaje y la lengua meta).

Igualmente, las estrategias influirían en la manera en que el aprendiz procesa la información (Mayer, 1988). Aplicado al campo de aprendizaje de LE, esto tendría consecuencias no sólo para el estudio del idioma, sino también para el aprendizaje derivado de la comunicación en interacción.

En lo que al aprendiz se refiere, la intencionalidad del uso de la estrategia es la característica principal (Faerch y Kasper, 1984b; Palmer y Goetz, 1988; Oxford, 1990). Rampillon (1985) la entiende como el propósito del aprendiz de aprender, el reconocimiento de las necesidades y del sentido del aprendizaje, y la decisión de no ser un obstáculo para su propio aprendizaje.

Esta característica conllevaría una activa inversión de esfuerzo por parte del alumno y provocaría que, si éste no percibe una compensación en el resultado logrado, rechace el uso de la estrategia aunque sepa que podría facilitarle el aprendizaje (Palmer y Goetz, 1988).

Del mismo modo, hay que indicar que las estrategias se relacionan con las diferencias individuales de los aprendices, lo que implicaría una variación en el uso según la persona. Aunque Ellis (1997) afirma que los resultados de los estudios empíricos son todavía demasiado débiles para explicar esta relación, todo apunta a que las diferencias individuales son un factor de influencia. En este sentido, Skehan (1989) señala la aptitud lingüística, la motivación y los factores cognitivos y afectivos (extroversión - introversión, asunción de riesgos, inteligencia, dependencia de campo y ansiedad); Larsen-Fremman y Long (1994) agregan la edad y el sexo; O'Malley y Chamot (1990) mencionan el grupo étnico o cultural de pertenencia; Oxford (1990) y Cohen (1998) incluyen el estilo de aprendizaje, mientras que Villanueva (1997) añade una relación con las representaciones que los individuos se hacen sobre sí mismos y sobre lo que aprenden.

De esta forma, conviene incidir en que las estrategias no son buenas o malas en sí mismas, sino en cuanto actúan de manera eficaz para los diferentes individuos, de acuerdo a su estilo personal de aprendizaje y a las características concretas de la tarea que deben resolver (Cohen, 2003).

Ateniéndonos al cuarto criterio de caracterización, la relación de las estrategias con otras variables del proceso de aprendizaje, destaca que las estrategias empleadas en contextos de enseñanza formal son diferentes a las surgidas en contextos extraacadémicos (Palmer y Goetz, 1988; Ellis, 1997). Esto podría deberse, entre otros factores, a que el uso de las estrategias en situaciones de instrucción es el resultado de una variedad de factores como el conocimiento previo, tanto del tema tratado en clase como del aprendizaje en sí, y el producto final o resultado que el profesor tiene en mente (Wenden, 1991: 30). De igual modo, el profesor juega un papel importante en las estrategias que los aprendices dicen usar 
(O’Malley y Chamot, 1990), mientras que las características de la tarea también influirían en la efectividad de las estrategias (Oxford, 2004).

Por último, en relación con la comunicación, cabe afirmar que las estrategias sirven al objetivo general de la competencia comunicativa (Oxford, 1990) y que se ponen en marcha tanto para la producción como para la recepción cuando el hablante no nativo considera que la comunicación corre peligro de interrumpirse (Bialystok, 1990).

Dadas estas características y tomando como base las definiciones de los diferentes autores, podemos concluir que las estrategias del aprendiz son planes de acción que regulan, controlan y evalúan los modos de actuación del aprendiente, se dirigen a un objetivo concreto de cara a optimizar los resultados de sus tareas de aprendizaje lingüístico, comunicación y procesamiento de la información de la lengua meta, y se orientan a fomentar el desarrollo personal del individuo como aprendiz y usuario de la nueva lengua.

\section{La transmisión didáctica de las estrategias}

\subsection{Posibilidad efectiva de la instrucción}

La posibilidad efectiva de la instrucción de las estrategias debe plantearse en torno a tres variables: en relación con la naturaleza de las estrategias como procesos cognitivos potencialmente conscientes; en referencia al contexto en el que se quiere llevar a cabo la instrucción, y respecto a la incidencia de las estrategias en el desarrollo del aprendizaje lingüístico. El uso de las estrategias es una tarea cognitiva compleja para el aprendiz, por lo que un intento de instrucción sólo se verá justificado si el empleo de las estrategias concretas seleccionadas puede enseñarse y aprenderse, aceptando que el aula de idiomas puede ser un contexto de aplicación real de las estrategias y que la instrucción de las mismas contribuye a una mejora del aprendizaje lingüístico.

La psicología cognitiva distingue entre los componentes declarativo, procesual y metacognitivo del conocimiento, entendiendo conocimiento como la información grabada en la memoria que es tanto requisito como resultado de las acciones y percepciones. Respecto al conocimiento lingüístico de manera específica, Faerch y Kasper (1984a) consideran que el componente declarativo lo forman las reglas de la interlengua y que se podría ordenar en los niveles gramatical, pragmático y discursivo, mientras que el procesual lo formarían los procedimientos de recepción, producción, interacción y aprendizaje, de los cuales, los tres primeros se dirigen a la comunicación $\mathrm{y}$, el cuarto, pretende establecer una parcela de conocimiento declarativo lingüístico mediante la interacción con los procedimientos de uso de la lengua. En cuanto al conocimiento lingüístico metacognitivo, nos estaríamos refiriendo al control dirigido de los procesos de apropiación y producción lingüística en los diversos ámbitos de uso de la lengua.

Dado que los tres componentes juegan un papel relevante en el aprendizaje y uso de la LE, parece sensato considerarlos parte de la competencia comunicativa, como hacen Faerch, Haastrup y Phillipson (1984), quienes mantienen que ésta puede referirse a la habilidad de hablar sin que el hablante tenga mucho conocimiento de los principios que subyacen a ese dominio, pero también puede corresponderse con el conocimiento de "ser capaz de comunicar" entendido como conocimiento "sobre" la comunicación. De este modo, si la competencia comunicativa es un objeto de enseñanza de LE, tendría que incorporar algo 
más que el dominio de la lengua, ya que los aprendices deberían desarrollar la conciencia metacomunicativa, es decir, el conocimiento consciente acerca de los componentes de la competencia comunicativa, su interdependencia y funciones sociales.

Por otra parte, la posibilidad de que el conocimiento procesual sea explícitamente enseñado y aprendido se basa en el grado de conciencia que implique el procedimiento en concreto. Tal y como se ha señalado anteriormente, las estrategias se pueden ejecutar de manera potencialmente consciente, por lo que, de acuerdo con O'Malley y Chamot (1990), podrían representarse como conocimiento procesual susceptible de adquirirse a través de estadios de aprendizaje cognitivo, asociativo y autónomo.

Desde el punto de vista de la enseñanza, como sostiene Knapp-Potthoff (1982), dado que dichos procedimientos pueden aislarse y son modificables por el propio aprendiz, se podría influir didácticamente en su adquisición y desarrollo mediante la manipulación de las condiciones bajo las cuales surgen y actúan.

Sin embargo, a pesar de las múltiples investigaciones que apoyan estas teorías, lo cierto es que carecemos de una base empírica lo suficientemente amplia como para afirmar que las estrategias pueden ser realmente enseñadas y aprendidas. A juicio de Dörnyei (1995), esta teachability controversy, como el autor la denomina, se sustenta en tres observaciones: que la mayoría de los argumentos tanto a favor como en contra de la instrucción se basan en la evidencia indirecta; que existe una variación entre las diferentes estrategias con respecto a la posibilidad de su enseñanza, y que la noción de enseñanza se deja interpretar de diversas maneras por los distintos autores.

Para salvar estos obstáculos, la instrucción de las estrategias se justifica tomando como base tres supuestos: la posibilidad de explicar el concepto y funcionamiento de las estrategias aplicadas al aprendizaje y la comunicación en LE, de acuerdo con las aportaciones de la psicología cognitiva; la posibilidad de demostrar visiblemente los efectos que causa la aplicación invisible de las estrategias concretas en la realización de tareas lingüísticas determinadas, a partir de los estudios de intervención que han empleado técnicas de modelado de las estrategias (O'Malley y Chamot, 1990; Harris y Gaspar, 2001; Macaro, 2001), y la posibilidad de ofrecer en el aula oportunidades de práctica guiada y libre en la aplicación de un amplio repertorio de estrategias aplicables a las tareas lingüísticas habituales, centrando la atención del aprendiz en el proceso de realización de la tarea más que en el resultado (Grenfell, 2002).

$\mathrm{El}$ interrogante que se plantea, entonces, para la práctica didáctica, es el modo de adecuar el aula para permitir la ejecución de las estrategias de forma modificable y susceptible de transferirse a futuras situaciones extraacadémicas. Así, las estrategias se presentan como un puente de unión para solventar las carencias y aprovechar las ventajas que la instrucción formal ha demostrado brindar al aprendizaje lingüístico.

De cara a la práctica en la clase de LE, convendría implantar tareas que inciten a los alumnos a avanzar en sus capacidades metacognitivas (de reflexión sobre el proceso de aprendizaje y sobre la ejecución actual de destrezas lingüísticas y comunicativas) y cognitivas (abstraer, generalizar, establecer analogías...), tareas que, por principio, exigen la aplicación de estrategias similares a las que se emplean en contextos reales de uso de la lengua. Otra ventaja del desarrollo de la dimensión metacognitiva en el aula es la contribución a la monitorización de la realización lingüística, entendida en términos de control, como hace Bialystok (1990) en su modelo de adquisición de segunda lengua. Según la autora, 
la habilidad de comunicar se alcanza mediante un equilibrio de los niveles de análisis del sistema lingüístico y control del procesamiento. De esta forma, la competencia estratégica consistiría en la habilidad de usar eficazmente el lenguaje para la comunicación a través de las estrategias basadas en el análisis y el control.

Los rasgos del modelo de Bialystok serían relevantes para distinguir entre competencia como conocimiento y como habilidad (Little, 1996). Al atribuir al control la función de selección del foco de atención adecuado, el modelo proporciona una explicación plausible del hecho de que la competencia estratégica opere algunas veces por debajo, y otras por encima, del umbral de la conciencia. Igualmente, el modelo requiere que el dominio lingüístico sea conceptualizado no como un fenómeno unitario, sino como una variedad de acuerdo con la actividad comunicativa concreta, por lo que la competencia estratégica debería definirse de nuevo para cada actividad, circunstancia ésta que favorece la acción didáctica al permitir que se determinen los diferentes parámetros de uso lingüústico y estratégico en función de las características de las tareas individuales.

Una vez aceptada la posibilidad de la transmisión didáctica de las estrategias, conviene aclarar los propósitos y metodología de la instrucción en el aula de LE para plantear, seguidamente, las premisas de integración de la competencia estratégica en el currículo.

\subsection{Propósitos y metodología didáctica}

La finalidad de la instrucción de las estrategias es, desde el punto de vista del enseñante, asistir a los aprendices en la mejora de las habilidades lingüísticas con un método sistemático que les ayude a desarrollar y refinar las destrezas de aprendizaje, por lo que el entrenamiento del individuo como "aprendiz" debería estar integrado en el entrenamiento lingüístico (Larsen-Freeman y Long, 1994: 197).

En efecto, los sucesivos proyectos de lenguas modernas del Consejo de Europa han enfatizado la importancia de, por un lado, habilitar al aprendiz para que actúe independientemente como usuario de la lengua meta y, por otro lado, capacitarle para que aprenda cómo aprender. El argumento central de este doble propósito es que el desarrollo de la autonomía de uso de la lengua extranjera está estrechamente relacionado con el desarrollo de la autonomía en el aprendizaje de la misma (Holec et alii, 1996). Consecuentemente, el Marco común europeo promueve la instrucción de la competencia estratégica sólo en el grado en que contribuya al control estratégico del aprendiz sobre sus procesos de aprendizaje lingüístico.

Por otra parte, desde el punto de vista del aprendiz, se pretende conseguir hacer por uno mismo aquello de lo que normalmente se ocupa el profesor en el aula. Harris (1997) se refiere a esta función como un método alternativo en la enseñanza de lenguas, centrado en capacitar a los aprendices para que se conviertan en más efectivos, de cara a que puedan compartir con el profesor la responsabilidad de sus progresos.

El establecimiento de una metodología adecuada a la transmisión de las estrategias, por otro lado, pasa por la consideración de los tres factores inherentes al contexto formal de enseñanza / aprendizaje de LE, esto es, la estructuración didáctica del input en el aula, la integración de los contenidos procedimentales en los lingüísticos, y el traspaso paulatino de responsabilidad del docente al alumno a medida que se desarrolla su capacidad metacognitiva. 
Así, si el contexto de adquisición natural posee la ventaja del input constante y de la actuación frecuente del hablante no nativo bajo determinadas condiciones de presión, en el contexto formal deberán suplirse estas aportaciones mediante la metodología, es decir, a través de la intervención metódica dirigida a una estructuración didáctica del input, previa al procesamiento o a la transformación de los datos lingüísticos por parte del aprendiz (Tönshoff, 1992: 22).

El incremento de las reservas disponibles de conocimiento lingüistico y el fomento de los procesos de automatización en el acceso a las mismas, se pueden lograr mediante el tratamiento de los datos lingüísticos de forma planeada, a través de diversos procesos de práctica en el aula. La enseñanza debe incluir tanto los procesos imitativos de habitualización (defendidos por las teorías conductistas de ASL) como los cognitivos, entendidos ambos como vías complementarias e interdependientes del proceso de aprendizaje de la LE.

\subsection{Condicionantes de la acción didáctica}

La cuestión de la efectiva contribución de las estrategias al proceso de aprendizaje debe, sin duda, abordarse con cautela, dado que, a pesar del optimismo de la mayoría de los estudios de intervención en el aula (Ellis y Sinclair, 1989b; O’Malley y Chamot, 1990; Oxford, 1990; Wenden, 1991; Graham, 1997; Chamot et alii, 1999; Harris y Gaspar, 2001; Macaro 2001), no puede obviarse que falta una constatación empírica que avale la instrucción (Rees-Miller, 1993; Ellis, 1997), principalmente, por la inexistencia de un instrumento capaz de medir la correlación entre la instrucción formal de las estrategias y el nivel de dominio lingüístico.

Por esta razón, aunque todo apunte a que pueda incidir positivamente en el desarrollo del aprendiz, un programa de LE que pretenda incluir explícitamente las estrategias debe tener en cuenta las reservas recogidas en la bibliografia, con el fin de amortiguar los efectos negativos que el tratamiento didáctico de la competencia estratégica podría originar sobre las demás.

Por un lado, las voces en contra de la instrucción en el uso de las estrategias (Bialystok, 1990; Kellerman, 1991; Rees-Miller, 1993) alegan que es un esfuerzo arduo que no conduce a una mejora segura del aprendizaje, ya que es innegable la evidencia de que el uso de las estrategias es un ajuste dentro del proceso de adquisición de segundas lenguas que no difiere del uso no estratégico del lenguaje. Más allá, señalan que algunas estrategias que emplean los adultos competentes podrían deberse sólo a su mayor dominio lingüístico, por lo que los docentes no deberían ocupar el tiempo de enseñanza con la instrucción en el uso de las estrategias, sino dedicarse a enseñar el lenguaje.

Los seis puntos que componen sus críticas se refieren a la escasa contribución de las actividades de concienciación al aprendizaje lingüístico, la necesidad de que el docente domine el uso y evaluación de las estrategias, la dificultad de adecuar la instrucción a las diferencias individuales de los aprendices, la disminución del tiempo dedicado a la práctica lingüística a favor de las estrategias, la transferencia natural de la competencia estratégica inconsciente de la lengua materna a la meta, y la falta de evidencias empíricas de la contribución a la mejora de la competencia lingüística.

Por otro lado, los estudios de intervención en el aula justifican la instrucción sistemática de las estrategias basándose en las ventajas que se derivan para el proceso de aprendizaje, sobre todo, para los componentes socioafectivo y metacognitivo. De acuerdo con sus resulta- 
dos, las estrategias mejoran la realización lingüística, tienen el efecto de fortalecer el aprendizaje, ayudan a aprender más rápido, apoyan el proceso de aprendizaje continuo, fomentan la individualización del proceso de aprendizaje, permiten que los aprendices se conviertan en más autodirigidos, favorecen la autonomía del aprendiz contribuyendo a su independencia y madurez, juegan un papel relevante para la supresión de los bajos rendimientos, dotan a los alumnos de un papel más activo en el proceso de aprendizaje, contribuyen a reducir el estrés, y ayudan a los individuos a convertirse en mejores aprendices, así como a desarrollar el control y la responsabilidad del propio aprendizaje.

\section{Modalidades de integración de la competencia estratégica en el currículo}

\subsection{El entrenamiento como método de instrucción}

De acuerdo con lo anteriormente expuesto, las premisas de estructuración didáctica del input, hábito y conciencia de la ejecución de los procesos de aprendizaje, más los propósitos de los participantes, conducen a un entrenamiento en el uso de las estrategias encaminado a implantar los procedimientos más efectivos para el procesamiento de un input que ha sido previamente estructurado de acuerdo a una situación de aprendizaje concreta, en la que el docente va delegando progresivamente la responsabilidad de la gestión del aprendizaje en el alumno de manera consciente para éste.

La finalidad de la metodología de instrucción es ofrecer continuadamente oportunidades y materiales de práctica, así como proporcionar un apoyo al aprendiz que se irá retirando poco a poco a medida que avance en su grado de dominio; un sistema de "andamiaje" o scaffolding que Macaro (2001: 175) define de la siguiente forma:

...scaffolding means supporting a course of action that we want learners to take by providing the means by which they are reminded to carry out that course of action, in a controlled way, with the objectives that it will become automatic and more autonomously applied once the learner judges that action to be efficacious for their learning or for carrying out a particular task.

El término mayoritariamente empleado para la metodología de instrucción de las estrategias es el de entrenamiento [training], adoptado en los estudios de adquisición de segundas lenguas porque, como indica Oxford (1990), es lo bastante descriptivo y general para servir a las necesidades específicas de esta área. Según la autora, entrenamiento hace referencia a la posibilidad de cambiar los hábitos y costumbres mediante la ejercitación de determinadas habilidades. A este respecto, Tönshoff (1992: 235) añade que se trata del término más adecuado para aplicarlo a la situación del aula, puesto que implica que la formación y los cambios en el conocimiento procesual del aprendiz no se producen por la mera presentación de instrucciones, sino que la práctica es de crucial relevancia.

La perspectiva del entrenamiento estratégico, según recogen Derry y Murphy (1986), emerge de la revisión de tres teorías: la teoría del entrenamiento de Gagné (1985), la teoría de la inteligencia de Sternberg $(1983,1985)$ y la teoría metacognitiva, en relación con los trabajos en el campo de la psicología cognitiva y del desarrollo (Brown, 1978; Flavell, 1979). 
Según la teoría de Gagné, la habilidad del aprendizaje es, en parte, una destreza intelectual entrenable y, en parte, una capacidad de pensamiento estratégico que debe implicar una función de experiencia e inteligencia. Las estrategias cognitivas serían procesos mentales superiores que se implantan para adquirir o acceder a los otros cuatro tipos de conocimiento (actitudes, destrezas motoras, información verbal y destrezas intelectuales), así como para organizarlos y usarlos en dirección a una meta.

Respecto a la segunda teoría, Sternberg presenta la habilidad del aprendizaje como una forma de inteligencia que tiene componentes entrenables. Para poner en práctica una estrategia, el aprendiz necesita acceso a un repertorio del que pueda escoger y, además, debe seleccionar los componentes o procedimientos individuales que posibilitan la ejecución de la estrategia e incorporarlos a la más adecuada para la situación de aprendizaje.

En cuanto a la teoría metacognitiva, se propone que el desarrollo de la habilidad de aprendizaje conlleva, por una parte, la capacidad de activar ciertos procesos de pensamiento y, por otra, la disponibilidad de un "almacén de memoria" que contiene un repertorio de las tácticas de aprendizaje, así como el conocimiento de su utilidad y uso. Una de las mayores contribuciones de la teoría metacognitiva ha sido resaltar el papel del conocimiento declarativo (explícito) de las estrategias en el control ejecutivo. Este conocimiento puede enseñarse de diversas formas, bien a través de la instrucción directa, bien mediante el uso correcto de tácticas entrenadas, o desde las experiencias metacognitivas conseguidas en el ambiente del entrenamiento.

Estas teorias nos recuerdan el carácter complejo y polifacético de la finalidad que queremos lograr con el entrenamiento de los alumnos en el uso de las estrategias: ampliar el punto de vista sobre su propio proceso de aprendizaje mediante oportunidades para seleccionar sus objetivos, reflexión sobre sus actitudes y preferencias como aprendices, experimentación con diferentes actividades de práctica y ejercitación de la auto-evaluación.

\subsection{Tipos de entrenamiento estratégico}

Según los estudios que sentaron las bases de la actuación empírica (Dansereau, 1985; Ellis y Sinclair, 1989b; Cohen, 1990; Oxford, 1990; Wenden, 1991; McDonough, 1995, entre otros), los modelos de entrenamiento para la ejercitación de estrategias en el aula difieren según sea su inclusión en el currículo, el grado de explicitud en la transmisión de las estrategias y la cantidad de información suministrada a los aprendices sobre el proceso de aprendizaje.

De esta forma, un modelo de entrenamiento podría ser incluido o excluido, dependiendo de si su implantación se realiza en el curso de lengua o se lleva a cabo en uno paralelo y separado, destinado exclusivamente al desarrollo de la competencia estratégica de los aprendices fuera del aula de idiomas; se habla de entrenamiento explícito cuando las estrategias se transmiten explicando cómo se llaman, en qué consisten, cómo se utilizan y cuál es su finalidad, mientras que el entrenamiento implícito consiste en fomentar su uso mediante tareas sin que el aprendiz sea consciente de que está poniendo en marcha un comportamiento estratégico; y puede efectuarse de manera informada, haciendo partícipes a los alumnos de las repercusiones del entrenamiento a lo largo de su trayectoria, o reservando esa información para el profesor o investigador. 
Para decidir el tipo de entrenamiento más adecuado a cada situación didáctica específica, conviene analizar las demandas respecto al grado de información, a la precisión en la explicación de las estrategias y a la inclusión en el currículo, que se derivan de las necesidades del grupo de aprendices objeto de la instrucción, ya que no todos los aprendices pueden o están dispuestos a asumir la responsabilidad sobre su proceso de aprendizaje en la misma medida debido a factores personales y de dominio lingüístico.

Los estudios más recientes, sin embargo, han puesto de manifiesto la necesidad de revisar estas teorías a la luz de los resultados obtenidos en las aulas, y la conveniencia de investigar más a fondo los condicionamientos óptimos del entrenamiento de las estrategias. Según sus conclusiones (Mazzotta, 1996; Graham, 1997; Harris, 1997; Grenfell y Harris, 1999; Harris y Gaspar, 2001; Macaro, 2001; Valiente, 2004), el entrenamiento informado, explícito e integrado en el currículo de LE es el que mayores posibilidades de éxito presenta, dado que fomenta el espíritu de cooperación entre el profesor y los alumnos, permite al individuo una auténtica toma de conciencia sobre su proceso de aprendizaje y favorece la transferencia de las estrategias aprendidas en el aula a futuras situaciones extraacadémicas, y justifica la inversión del tiempo dedicado a las estrategias al enlazar los contenidos lingüísticos con los procedimientos más efectivos para el procesamiento y uso del lenguaje.

\section{Pautas para una integración explícita e informada de la competencia estratégica}

Respecto a las recomendaciones de las instituciones que coordinan las líneas de acción en el campo de la enseñanza / aprendizaje de idiomas, el Marco común europeo de referencia para las lenguas no indica ninguna opción como la más favorable para la instrucción de las estrategias en el aula, si bien deja a la libre decisión del usuario un amplio abanico de posibilidades que van desde la creación de situaciones y la realización de tareas que requieran operaciones y estrategias de planificación, ejecución, evaluación y corrección, hasta animar o exigir a los alumnos que sigan procedimientos estratégicos explícitos cuando sean necesarios, pasando por la ejercitación de técnicas de reflexión y toma de conciencia.

En lo tocante a la enseñanza del español, el Plan Curricular del Instituto Cervantes (1994) incluye la transmisión de las estrategias de aprendizaje y comunicación como uno de sus fines generales, y considera que pueden acompañar al proceso de aprendizaje tanto de forma espontánea e inconsciente como de manera consciente y programada; sin embargo, no se pronuncia sobre el mejor modo de llevar a cabo la instrucción.

El reto al que se enfrentan los investigadores es determinar la forma en la que pueden ejercitarse las estrategias dentro del programa habitual del curso sin apartarse de las actividades de práctica lingüística ni consumir un tiempo excesivo en el fomento de destrezas procedimentales que se alejen del uso de la lengua. Veámos los criterios de base para ello.

\subsection{Fundamento teórico}

El profesor debe conocer la manera en que los aprendices procesan la información de cara a decidir las variables externas más apropiadas para apoyar y sostener los procesos internos del aprendizaje, por lo que se hace necesaria una teoría del procesamiento de la 
información que justifique las decisiones adoptadas. La necesidad de un fundamento teórico para el modelo de entrenamiento es uno de los aspectos que ponen de relieve los estudios de intervención (Grenfell y Harris, 1999).

Los diferentes métodos de entrenamiento tienen un impacto distinto en las actitudes y comportamientos de los aprendices. Las conclusiones de los estudios anteriores pueden ilustrar al creador del modelo de entrenamiento acerca de la relación entre las estrategias y las perspectivas teóricas existentes, los efectos de entrenamiento a largo plazo, y la posibilidad de integrar el entrenamiento en los cursos regulares de $\mathrm{LE}^{3}$.

\subsection{Enfoque, amplitud y profundidad del entrenamiento}

El enfoque viene determinado por las decisiones de integración, explicitud e información. Como ya se ha expuesto anteriormente, todo indica que el único entrenamiento susceptible de éxito es el informado, explícito e integrado en el aprendizaje lingüístico.

Respecto a las decisiones restantes, la amplitud corresponde a las destrezas lingüísticas y sociolingüísticas que se verán favorecidas por el entrenamiento, mientras que la profundidad se refiere a la cantidad de apoyo que se concederá a la destreza en cuestión mediante la aplicación de las estrategias, es decir, si sólo se facilitará su desarrollo mediante el uso de estrategias cognitivas (que afecten directamente a la manipulación del input lingüístico) o bien se complementará la ejecución de éstas con el ejercicio de otras de carácter metacognitivo y socioafectivo.

De esta forma, el modelo puede orientarse a entrenar las estrategias necesarias para el desarrollo de las cuatro habilidades tradicionales (comprensión lectora, comprensión oral, expresión escrita y expresión oral) o sólo a una de ellas, así como favorecer el aprendizaje de reglas gramaticales o de adquisición de léxico. Para ello, puede seleccionar estrategias que afecten directamente a la manipulación del input lingüístico (estrategias de resumen, de toma de notas, de memorización, de asociación de imágenes y sonidos...) o combinar éstas con otras que contribuyan a la planificación y evaluación del aprendizaje, la motivación del individuo, la asunción de riesgos, etc.

Ambas variables, amplitud y profundidad, dependen, en última instancia, del objetivo final del curso de LE y serán más estrechas cuanto más concretas sean las necesidades de los aprendices.

Con respecto a la profundidad, las dimensiones de aprendizaje que deben incluirse, puede afirmarse que las llamadas estrategias indirectas (metacognitivas y socioafectivas) contribuyen a un mejor empleo de las cognitivas (Graham, 1997; Valiente, 2004), así como a un incremento de la motivación y al logro de mayor autonomía en el proceso de aprendizaje (McCombs, 1988; Wenden, 1991).

3 Respecto a la relación entre las estrategias y las perspectivas teóricas, O’Malley y Chamot (1990) ofrecen un marco basado en la teoría del procesamiento de la información de Anderson que puede resultar de utitidad. Para más información sobre la integración del modelo en un curso regular de lengua, el ejemplo más claro lo encontramos en Chamot et alii (1999). Acerca de los efectos del entrenamiento a largo plazo, sin embargo, los resultados son poco concluyentes, dado que los últimos estudios de intervención consideraron los siete meses disponibles como tiempo insuficiente (Harris y Gaspar, 2001). 


\subsection{Adecuación a las necesidades de los aprendices}

Las necesidades de los alumnos afectan a la amplitud y profundidad del entrenamiento, a la selección de las estrategias concretas que se van a ejercitar, y al diseño de las tareas y actividades de elicitación de estrategias.

Respecto a su incidencia en la amplitud, en el caso de que el entrenamiento esté centrado en una destreza lingǘstica en la que el alumno muestra una especial carencia de recursos, conviene determinar las necesidades reales de empleo de la destreza en cuestión. Por ejemplo, un entrenamiento orientado a la comprensión oral en el país de la lengua meta, atenderá especialmente a las estrategias de petición de aclaración, repetición mental de sonidos, reelaboración en voz alta del mensaje del interlocutor y control de las emociones, mientras que, si esa misma destreza va a usarse en el país de origen en un contexto académico, será más útil practicar las estrategias de inferencia, reconocimiento de la estructura del texto, localización de la información principal, toma de notas y resumen.

En cuanto a la profundidad, dependerá del nivel de autonomía requerido por la situación de los aprendices y de la cantidad de responsabilidad que son capaces o están dispuestos a asumir; si necesitan desenvolverse como usuarios autónomos de la nueva lengua porque estén en continuo contacto con ella, precisarán la aplicación de estrategias metacognitivas de monitorización de la propia realización lingüística, así como de otras socioafectivas dirigidas a la motivación y la incentivación a asumir riesgos. Si, por el contrario, el uso de las estrategias está destinado a reforzar el aprendizaje asistido por un docente en el contexto académico, las estrategias de planificación y evaluación pasarán a un segundo plano a favor de las de manipulación del input lingüístico, debido a las menores exigencias impuestas por la situación comunicativa.

Con relación a la influencia de las necesidades de los aprendices en la selección de las estrategias concretas, se debe evitar entrenar a los alumnos en las que ya conocen o en aquéllas que sean menos eficaces que las que ya utilizan (Peterson y Swing, 1983). Asimismo, conviene ceñirse a las que se puedan entrenar en el tiempo de duración del curso de lengua (Derry y Murphy, 1986) y que sean susceptibles de transferirse a las situaciones reales de aplicación que necesita el nuevo hablante.

A la hora de diseñar las actividades de elicitación de estrategias, la condición fundamental es la relevancia para el aprendizaje lingüístico, es decir, la coincidencia con los objetivos de los aprendices porque, si no encuentran que las estrategias sean significativas para su aprendizaje, no las usarán (Wenden, 1991). De este modo, las necesidades de los aprendices determinarán que el entrenamiento se oriente a la resolución de actividades similares de una tipología específica para facilitar una rápida transferencia (como sería en el caso de un curso preparatorio de un examen oficial), o a tareas de características diferentes que presentan mayor dificultad a la hora de escoger, aplicar y transferir el uso de las estrategias (en el caso de un curso de aprendizaje lingüístico general).

\subsection{Atención a las diferencias individuales}

Afectan a la planificación del entrenamiento en la selección de las estrategias que van a enseñarse, en la elección de los aprendices que se someterán al entrenamiento de cada una de ellas (en el caso de que no todas sean adecuadas para el grupo completo) y en el diseño de las actividades. 
La inclusión de estos factores comienza por recabar información indicativa de cómo las diferencias individuales afectarán al entrenamiento, lo que permitirá, por un lado, prever las acciones necesarias para paliar los efectos negativos en el caso de que se produzca un choque entre los factores personales y el tratamiento didáctico y, por otro lado, posibilitará que se logre el máximo rendimiento del potencial del individuo.

Los investigadores (Ellis y Sinclair 1989a; Oxford, 1990; O'Malley y Chamot, 1990; Wenden, 1991) recomiendan el empleo de cuestionarios ${ }^{4}$ en la sesión o sesiones introductorias para detectar los siguientes factores personales: nivel de dominio lingüístico; experiencia en el aprendizaje de LE y el uso de estrategias; estilos de aprendizaje y bagaje educativo y cultural del aprendiz; motivación, actitudes y creencias.

\subsection{Finalidad y objetivos del entrenamiento}

En un programa de instrucción de enfoque restringido, la finalidad se puede establecer a partir de una destreza lingüística, de los puntos débiles de los aprendices o de las necesidades específicas impuestas por el curso de idiomas. Así, un modelo de entrenamiento podría tener como finalidad última el logro de una mayor autonomía en la resolución de tareas de una sola destreza lingüística (por ejemplo, conseguir fluidez en la expresión oral); el dominio de una o más estrategias de uso generalizable a varias o todas las destrezas (estrategias de razonamiento deductivo y transferencia del conocimiento previo para la realización de tareas de las cuatro destrezas); la resolución de un problema concreto de uso de la lengua mediante el empleo de estrategias que afectan a una o más dimensiones del aprendizaje (consecución de una mejora de la comprensión auditiva intensiva en las situaciones de comunicación en las que el oyente no tiene posibilidad de interacción), o la autonomía en la realización de una tarea lingüística concreta que requiere unas estrategias específicas (redacción de trabajos científicos para la Universidad).

La finalidad servirá como punto de referencia para determinar la manera en que se pretenden alcanzar los objetivos individuales perseguidos con el entrenamiento, es decir, que orientará la selección de las técnicas de instrucción y la elaboración de la taxonomía de estrategias.

Respecto a los objetivos específicos, las investigaciones no ofrecen una formulación explícita y, cuando lo han hecho, normalmente ha sido en forma de etapas de las sesiones del entrenamiento (como es el caso de Wenden, 1991: 104).

El procedimiento más útil pasa por la distinción de la finalidad, entendida como la formulación de la pretensión más amplia del entrenamiento; los objetivos, concebidos como la expresión concreta de las aspiraciones del programa, y las estrategias, que son el medio para cumplir estas aspiraciones y conseguir la finalidad pretendida. Esta distinción es necesaria, por una parte, para evitar el riesgo de confundir los objetivos con las etapas del entrenamiento y, por otra, con el fin de diferenciarlos de la taxonomía de estrategias para no desvirtuar la finalidad última de la instrucción, ya que las estrategias que se van a ejercitar no son un objetivo en sí, sino un medio para lograr una finalidad más compleja.

4 En las obras citadas se ofrecen muestras de cuestionarios de diagnóstico de las distintas variables, que el docente puede adaptar fácilmente a su grupo de aprendices. 
La determinación de los objetivos, por definición, sirve como base para la selección de tareas, el diseño de actividades y la elección de materiales. Para evitar incurrir en una confusión entre objetivos y etapas, u objetivos y estrategias, se propone a continuación un planteamiento genérico de los objetivos, generalizable a cualquier modelo de entrenamiento, de cara a que el responsable del programa los oriente - mediante la elección de tareas y el diseño de las actividades - a la finalidad última de su instrucción.

El planteamiento de objetivos se basa en las enunciaciones de Oxford (1990: 201) y Cohen (1998: 70-71) y resulta de la siguiente forma:

- Despertar el interés de los alumnos por conocer el funcionamiento de sus procesos de aprendizaje.

- Motivarlos a intervenir en el desarrollo de sus procesos de aprendizaje mediante el empleo de estrategias.

- Elicitar las estrategias que los alumnos ya utilizan para reflexionar sobre los recursos con los que cuentan y la manera de movilizarlos más eficazmente.

- Presentar una amplia taxonomía de estrategias adecuada a las necesidades de los aprendices.

- Proporcionar oportunidades de práctica guiada y supervisada para la ejecución en el aula de las estrategias seleccionadas.

- Incentivar y facilitar la puesta en práctica de las estrategias en situaciones de comunicación externas al marco del entrenamiento.

\subsection{Etapas del entrenamiento}

Todo modelo consta de más fases que la mera ejercitación de las estrategias, puesto que también incluye la preparación y la evaluación.

A pesar de que no existe una evidencia empírica que determine el mejor modo de conducir el entrenamiento (Cohen, 1998), la bibliografia ofrece varios marcos de instrucción ${ }^{5}$ que pueden ayudar al diseñador del programa a establecer la secuencia de los contenidos. Todos coinciden en tres grandes etapas que se exponen a continuación.

\subsubsection{Etapa inicial}

Respecto a la etapa inicial, Ellis y Sinclair (1989b: 7) identifican tres áreas que corresponden a la preparación psicológica, la metodológica y la práctica en la auto-gestión del aprendizaje. El modelo CALLA (Cognitive Academic Language Learning Approach) de O’Malley y Chamot (1990), por su parte, se orienta a la activación del conocimiento previo

5 Cohen (1998: 71) ofrece varios marcos: el de Pearson y Dole (1987), para Ll; el de O'Malley y Chamot (1990: 201), para aprendizaje de L2 en el país de la lengua meta pero aplicable a L2 en el país de origen, como se puede ver en la adaptación de Chamot et alii (1999: 46), y el de Oxford (1990: 204). Tönshoff (1992: 278) añade el modelo de Hosenfeld et alii (1981) de entrenamiento integrado de una única destreza en LE. Una progresión alternativa la ofrecen Ellis y Sinclair (1989b: 11-12) a partir de la superposición de etapas y destrezas lingüísticas. Basándose en la secuencia tradicional, Harris (1997) propone el Strategy Training Cycle, reformulado como Strategy Instruction Cycle (SIC) en Grenfell y Harris (1999) y Harris y Gaspar (2001), para educación secundaria. El Learner Strategies Training Cycle de Macaro (2001: 176) tiene la misma base pero un desarrollo más amplio. 
de los aprendices, al avance de la organización de la lección y a la atención selectiva de palabras y conceptos clave que se van a presentar, es decir, está más centrada en la preparación metodológica de Ellis y Sinclair que en la psicológica. En cuanto a Oxford (1990), la autora establece una preparación anterior incluso a la de los aprendices, que implica tan sólo al responsable de conducir el entrenamiento. El paso inicial se ocuparía de determinar las necesidades de los aprendices y el tiempo disponible, así como de seleccionar una taxonomía de estrategias e incluir el entrenamiento en el curso de aprendizaje lingüístico. A partir de aquí, sería necesario considerar la motivación de los alumnos, es decir, la mencionada preparación psicológica.

\subsubsection{Etapa de ejercitación}

Los diferentes programas de instrucción de las estrategias coinciden en los pasos que conforman esta etapa, si bien varían en la denominación otorgada. A grandes rasgos, todos pasan por las fases de modelado de la estrategia por parte del profesor / entrenador, oportunidades de práctica guiada en la aplicación de la estrategia, evaluación del uso de la estrategia para la realización de la tarea, actividades de práctica libre para perfeccionar la aplicación de la estrategia, evaluación de la selección y ejecución libres de la estrategia, y presentación de actividades similares (de expansión) para favorecer la transferencia.

Respecto a la fase de modelado, conviene distinguir entre varias posibilidades, a saber, la presentación verbal de la estrategia, su etiquetado, la demostración de su empleo y el recordatorio de su posible aplicación para resolver tareas concretas. El docente decidirá cuál es la más apropiada a la situación del grupo y de acuerdo con las características de cada estrategia, dado que un modelado exhaustivo de todas las estrategias susceptibles de ser aplicadas llevaría demasiado tiempo y sobrecargaría la capacidad y el interés de los aprendices.

En cuanto a las oportunidades de práctica guiada, conviene que se alternen las fases de presentación / modelado / recordatorio de uso de estrategias "nuevas" con las fases de práctica de las estrategias "antiguas". Así se posibilita la elección de la más adecuada para cada individuo, la aplicación de estrategias diferentes para una misma tarea, el empleo de estrategias en combinación y el refuerzo de uso de estrategias aprendidas tiempo atrás.

Del mismo modo, sería recomendable ofrecer comentarios de feedback como refuerzo a lo largo de la primera ocasión de práctica (Dansereau, 1985), por un lado, de cara a que los aprendices entiendan cuándo y cómo usar la estrategia y, por otro, para asegurar que han comprendido las explicaciones previas.

A continuación, se realizaría una evaluación inicial dependiente de los aprendices al finalizar cada actividad de práctica guiada y antes de las oportunidades de práctica libre (Macaro, 2001). No obstante, el docente debería comprobar simultáneamente que el alumno ha ejecutado las estrategias correctamente.

En cuanto a la presentación de las tareas para aplicar las estrategias de manera libre, el docente debe responder a tres cuestiones: si se ofrecerán tareas específicas para estrategias determinadas, si las tareas serán siempre similares o se cambiará de tipo, y la cantidad de práctica que se ha de ofrecer.

Del mismo modo que la práctica libre debe ir precedida de la guiada, la evaluación del uso autónomo también requiere del apoyo inicial del profesor (O'Malley y Chamot, 1990; 
Harris, 1997). Se trata, sin embargo, de una labor compleja que sólo pueden acometer los aprendices con una conciencia metacognitiva desarrollada, ya que exige, por una parte, distinguir la evaluación de la realización de la tarea lingüística y del uso de las estrategias $\mathrm{y}$, por otra, la verbalización de la evaluación. Es por ello que podría considerarse uno de los últimos propósitos del modelo de entrenamiento, necesaria, no obstante, para favorecer la autonomía del alumno en ocasiones extraacadémicas de uso lingüístico.

Estas oportunidades de práctica libre, por otra parte, deben distinguirse de las llamadas de expansión, encaminadas a favorecer la transferencia de las estrategias ejercitadas anteriormente a nuevas situaciones de uso de la lengua. Así, mientras las actividades de práctica libre son similares para favorecer el empleo rutinario de las estrategias, las de expansión pretenden la aplicación a una tarea nueva de las estrategias que el aprendiz ya domina.

\subsubsection{Etapa de evaluación}

Para llevar a cabo la evaluación, Tönshoff (1992: 277-278) distingue dos posibilidades. Por una parte, como responsabilidad del profesor, realizada a partir de los datos recabados antes y después del entrenamiento (mediante un test de dominio de las diferentes destrezas lingüísticas, por ejemplo) y, por otra parte, efectuada de manera consciente y conjunta entre todos los participantes para analizar y valorar los resultados del entrenamiento, con lo que constituiría una parte independiente del programa de entrenamiento.

El estudio de intervención de Macaro (2001) también recoge dos momentos en la etapa de evaluación: una inicial de los estudiantes inmediatamente posterior a las actividades de práctica guiada, y otra conjunta con el profesor después de las actividades de expansión. El interés de esta propuesta radica en que fomenta la conciencia metacognitiva de los estudiantes al mismo tiempo que los implica activamente en la valoración de la utilidad del tratamiento didáctico que se les está administrando ${ }^{6}$. Sin embargo, resulta oportuno añadir la conveniencia de que esta evaluación inicial sea supervisada por el docente de cara a ofrecer comentarios de feedback que pudieran ser necesarios para ayudar a los aprendices menos autónomos a autoevaluarse correctamente, así como a obtener información sobre su motivación y actitudes respecto al entrenamiento.

\subsection{Consideración de las condiciones espacio - temporales}

Las limitaciones temporales no impiden la instrucción de las estrategias, pero condicionan el tipo de entrenamiento, así como su enfoque y profundidad, y el número de estrategias seleccionadas.

La distinción más frecuente y generalmente aceptada por los investigadores es la del entrenamiento a corto y a largo plazo, una diferenciación basada en el número de estrategias, en las oportunidades de práctica y, por tanto, en las posibilidades de monitorización y autoevaluación del uso estratégico.

Sin embargo, la clasificación del entrenamiento que encontramos en la bibliografía en cuanto a la extensión temporal es arbitraria y vaga, dado que no hay una especificación de 
lo que se considera un plazo "corto" y uno "largo", mientras que se confunde la aplicación del término con la duración del curso de idiomas.

Para solventar este obstáculo, cabría preguntarse si la denominación de "plazo" se aplica al tiempo concedido a analizar los efectos del entrenamiento, o a la duración del curso de LE. Si nos referimos a la primera posibilidad, entonces un entrenamiento a largo plazo sería aquél que evalúa sus resultados al final del curso (es decir, comprende todo el tiempo disponible para el aprendizaje lingüístico), mientras que uno a corto plazo mediría la contribución del entrenamiento en un espacio temporal fijado de antemano y de inferior duración al curso de LE.

De esta forma, se establece una diferencia entre la duración del curso de lengua y el tiempo destinado a que el entrenamiento dé sus frutos, diferencia que parece necesaria si pretendemos que la competencia estratégica esté presente en cualquier programa de enseñanza / aprendizaje formal puesto que, de lo contrario, deberíamos excluirla de los cursos que no tuvieran una determinada duración mínima que ni siquiera está esclarecida por las investigaciones.

Respecto a las limitaciones impuestas por el contexto físico en el que se efectúa la instrucción, éstas afectan a la taxonomía de estrategias objeto del entrenamiento y al diseño de las tareas de aprendizaje.

En primer lugar, conviene considerar que los aprendices ponen en práctica estrategias diferentes en el aula que en situaciones reales de uso de la lengua (Ellis, 1997), por lo que será preciso seleccionar aquéllas que realmente se puedan ejercitar en el marco de la clase de idiomas. Por otra parte, a la hora de diseñar las tareas se deben prever los posibles efectos negativos que las dimensiones y características del aula pueden causar en lo que Nunan (1989:92) llama modo de trabajo (individual, en parejas o en grupos), y en la elicitación de las estrategias. Por ejemplo, una clase pequeña y con las mesas unidas en filas no favorecerá la puesta en práctica de estrategias de interacción en conversaciones de grupo, mientras que las condiciones de comprensión audiovisual en una sala demasiado grande con una pantalla pequeña serán mucho más duras que las que normalmente ofrece esta tarea en la vida real, lo que incrementará la dificultad de usar estrategias transferibles a futuras situaciones extraacadémicas.

\subsection{Identificación de las técnicas de instrucción}

Se pueden distinguir diferentes opciones de entrenamiento según la metodología empleada. Las seis que cita Cohen (1998: 74) son el entrenamiento general de destrezas de estudio separado de la clase de LE; a través de charlas informativas y talleres de práctica; mediante el trabajo guiado por un tutor; insertando discusiones sobre estrategias directamente en los manuales del alumno; mediante minicursos en formato vídeo, y el entrenamiento totalmente integrado en el currículo de LE bajo la dirección del profesor.

De todas ellas, la única susceptible de aplicarse al contexto tradicional de aprendizaje formal de LE, es la última. Para llevarla a cabo, Cohen menciona tres posibilidades: emplear los materiales previamente establecidos para el curso y determinar las estrategias que se van a insertar, así como el momento más adecuado para introducirlas; la instrucción lingüística a partir de un conjunto de estrategias seleccionadas por el docente para diseñar actividades alrededor de ellas, y la inserción de estrategias espontáneamente dentro de la lección allí donde parezca indicado. 
De cara a obtener una óptima integración de la competencia estratégica en el programa de LE sin que ésta se convierta en el eje vertebrador del mismo y, al mismo tiempo, considerando el esfuerzo que supondría para el docente la inserción de estrategias de manera espontánea para actividades planteadas de antemano sin tener en cuenta el componente estratégico, todo parece indicar que la instrucción se realizará más efectivamente de acuerdo con la primera posibilidad citada por el autor.

\subsection{Aclaración de los papeles del profesor y de los aprendices}

Quizá el requisito más necesario para llevar a cabo el entrenamiento estratégico sea el cambio de mentalidad de los alumnos y el profesor acerca de los papeles que, tradicionalmente, han desempeñado unos y otro en el contexto de aprendizaje formal.

-El cambio de actitudes del docente se sitúa al mismo nivel de importancia que el conocimiento que debe tener de las estrategias (Oxford, 1990: 201) dado que, si no reestructura sus ideas y creencias sobre las funciones tradicionales, no logrará que los estudiantes asuman más responsabilidad sobre su propio proceso de aprendizaje.

El papel del profesor-entrenador debe incluir las facetas de responsable del diagnóstico de necesidades y estrategias, entrenador de nuevas estrategias, animador, coordinador, aprendiz de lenguas e investigador (Cohen 1998).

Respecto al mejor modo de cambiar la mentalidad de los alumnos, Wenden (1991: 126) recomienda la comunicación persuasiva, consistente en presentar explícitamente hechos que demuestren lo que los aprendices pueden hacer por sí mismos para ayudarse a aprender con éxito sin la ayuda del profesor, de manera que se transmita implícitamente el argumento de que son capaces de asumir más responsabilidad.

Uno de los componentes en el que debería producirse este cambio es, según Carver (1984), la evaluación. En este sentido, los aprendices deberían decidir — siguiendo unos criterios predeterminados - su propio trabajo y el de sus compañeros, y realizar la parte de la tarea decidida sobre la base de ese criterio; llevar un control de sus propios progresos y pedir confirmación al profesor de su autoevaluación; usar sus anotaciones de autoevaluación para seleccionar los materiales de revisión y solución de problemas, y constituir grupos de ayuda para resolver las dificultades de los miembros del grupo. En resumidas cuentas, la instrucción de las estrategias debe propiciar el aprendizaje colaborativo (Grenfell y Harris, 1999).

\subsection{Consideración de los materiales disponibles}

Un caso de condicionamiento de la labor docente por el material sería la obligatoriedad de emplear un determinado manual. En este caso, si no resultara lo suficientemente flexible como para favorecer el empleo de las estrategias, debería reforzarse con materiales complementarios o, como indica Oxford (1990: 207), con una guía de apoyo sobre el modo y el momento más adecuados para poner en práctica las diferentes estrategias.

$\mathrm{Si}$, por el contrario, el docente tiene total libertad para seleccionar el manual y/o los materiales, conviene contar con las limitaciones de oferta del centro educativo en cuanto a medios audiovisuales, recursos informáticos, fondos bibliográficos, etc., que podrían ser necesarios para llevar a cabo las tareas deseadas. 
En cuanto a la adecuación al estilo de aprendizaje de los alumnos, por una parte, es conveniente que no se produzca una actitud de rechazo ante materiales poco familiares o que presentan una dificultad de uso añadida a las propias de la tarea lingüística, así como que se adecúen al estilo cognitivo de los estudiantes, aunque podría resultar beneficioso añadir otros que desarrollen las facetas más débiles (Mazzotta, 1996).

\subsection{Actividades de elicitación y fomento de las estrategias}

Las actividades estratégicas se diferencian del resto de ejercicios destinados al aprendizaje lingǘstico en tres rasgos: su enfoque en el proceso más que en el contenido, el grado de información suministrada por el docente acerca de su contribución al desarrollo del aprendizaje, y la reflexión como resultado de su realización, a veces combinada con la experimentación (Ellis y Sinclair, 1989b: 17). De acuerdo con Fernández (2004: 874),

Lo importante es detenerse en los procesos que tienen lugar en la comunicación lingüística y en la apropiación de los recursos para diseñar desde ahí las actividades didácticas que activen los mecanismos o estrategias de comunicación y de aprendizaje.

Por otra parte, siguiendo la recomendación de Tönshoff (1992: 307), la condición de partida de tales actividades sería su estrecha relación con el grado de complejidad cognitiva que ofrecería una situación real (no académica) de uso de la LE, como, por ejemplo, mediante actividades comunicativas en forma de juegos de interacción o ejercicios de simulación.

Como consejos adicionales, cabe añadir que las actividades sean interesantes para los estudiantes (Oxford, 1990; Wenden, 1991), que reflejen el mundo real y se adapten al contexto de la clase (Nunan, 1989) y que resulten familiares al docente y a los alumnos, de cara a favorecer su aceptación y facilitar su resolución.

\subsection{Elaboración de una taxonomía de estrategias}

Las investigaciones recomiendan la selección de más de una estrategia, aunque se trate de un entrenamiento de enfoque limitado, la inclusión de estrategias que afecten tanto al nivel cognitivo del uso lingüístico como el metacognitivo de evaluación y control del aprendizaje, y la integración de estrategias socioafectivas que fomenten la motivación y la toma de riesgos. En cuanto a la elección de las estrategias según su aportación al aprendizaje, si bien es preciso entrenar en aquéllas que contribuyen específicamente a una mejora en el uso de la LE, no se descarta la introducción de otras más generales que puedan favorecer la memorización de ítems y reglas lingüísticas.

Paralelamente, Tönshoff (1992) señala que la elaboración o selección de una taxonomía de estrategias debe tener presente otros condicionamientos derivados del contexto de la instrucción y de los participantes: el objetivo general de aprendizaje de la clase de LE, la situación de aprendizaje, las necesidades de los aprendices, las características del grupo de estudiantes, la disponibilidad de materiales didácticos adecuados, y la compatibilidad con las estrategias de enseñanza y el estilo didáctico del profesor.

En consecuencia, el responsable del entrenamiento tiene la tarea de elaborar su propia taxonomía tomando estas consideraciones y requisitos como base de selección de las 
estrategias concretas para sus necesidades específicas, un trabajo que se ve enormemente facilitado gracias a las investigaciones anteriores ${ }^{7}$, que se han ocupado extensamente de la categorización, clasificación y descripción de las estrategias particulares, así como de su aplicación a las diferentes destrezas lingüísticas y tareas de aprendizaje.

\section{Conclusión}

De acuerdo con Villanueva (1997), en la concepción actual de la expresión enseñanza / aprendizaje en el aula, enseñar una lengua va apareciendo progresivamente como un trabajo de diagnóstico de estrategias de aprendizaje, como una evaluación de los sistemas lingǘsticos intermedios por los que atraviesa el aprendiz y como un diseño de situaciones de aprendizaje.

La pretensión del uso de estrategias en contextos de enseñanza formal surge de la necesidad de convertir el aula en un ámbito de aprendizaje que responda a las necesidades reales presentes y futuras de los aprendices como usuarios de una lengua extranjera y, como indica McDonough (1999), de llenar el vacío entre lo que los aprendices pueden hacer y lo que hacen.

Dado que la instrucción en el uso de las estrategias no es, sin embargo, una fórmula mágica para mejorar la realización de los aprendices (Chamot y Rubin, 1994), podemos afirmar que el componente estratégico no debe convertirse en el principal objetivo del aula de idiomas, ni en el eje vertebrador del programa del curso, sino integrarse mediante una metodología de transmisión del conocimiento procesual, es decir, de los procedimientos que facilitan la práctica de las destrezas de comprensión y expresión oral y escrita. Si bien esta necesidad no debe sustituir a la enseñanza del conocimiento lingüístico declarativo, tampoco puede obviarse en la labor docente, más bien ha de realizarse como un complemento de las competencias gramatical y sociolingüística, a cuyo desarrollo contribuye.

Dada la falta de investigaciones empíricas cuantitativas con conclusiones estadísticamente significativas, no estamos en situación de afirmar que la instrucción en el uso de las estrategias se traduzca en una mejora de la competencia lingüística. Las aportaciones demostradas hasta el momento parecen, sin embargo, lo bastante prometedoras como para aceptar que el efecto es más positivo que negativo, siempre que esté integrada en el programa de aprendizaje lingüístico y no se sitúe la ejercitación de las estrategias por encima de la práctica lingüística.

Por otra parte, la inclusión del componente estratégico requiere un esfuerzo extra del docente, puesto que tanto los objetivos y la metodología de la ejercitación de las estrategias, como el repertorio elegido para aplicarlo a las oportunas actividades de práctica lingüística, deben partir de la consideración de los diversos factores de influencia propios de cada situación de enseñanza / aprendizaje.

7 En Oxford (1986) encontramos una revisión de las taxonomías anteriores y una clasificación según la contribución directa o indirecta de las estrategias al aprendizaje lingüistico. La misma autora (1990: 317 y sigs.) ofrece una larga lista de estrategias aplicables a las distintas destrezas lingüísticas (Strategy Applications Listed According to Each of the Four Language Skills). Para un repertorio de estrategias clasificadas según las dimensiones cognitiva, metacognitiva y socioafectiva del aprendizaje, véase O'Malley y Chamot (1990: 137 y sigs.). En cuanto a las estrategias por destrezas, Harris (1997) ofrece una taxonomía resumida de sencilla aplicación a la práctica didáctica. 
Del mismo modo, el desarrollo consciente de la competencia estratégica orienta la acción didáctica a un enfoque en el proceso más que en el resultado, esto es, a la observación de los mecanismos que el alumno pone en marcha para resolver las tareas de uso lingüístico, a la elicitación de aquéllos que puedan contribuir positivamente a su resolución y a la evaluación constante de los procesos que subyacen a la actuación del aprendiz, lo que no siempre resulta viable.

Esta dificultad de la transmisión de las estrategias intrínseca a su condición de conocimiento procesual, se ve, además, incrementada por la inexistencia de unas pautas de aplicación didáctica inmediata generalmente aceptadas y respaldadas empíricamente, por lo que el docente ha de buscar y adaptar de la bibliografia un modelo de entrenamiento que se adecúe a las necesidades de su grupo de aprendices, una labor para la que a veces cuenta con referencias insuficientes.

Las líneas de actuación esbozadas en el presente artículo han recogido las consideraciones de los estudios de intervención para exponer, a modo de secuencia, las recomendaciones que afectan a las distintas variables que intervienen en el contexto de aprendizaje formal. En este sentido, se promueve una integración de la competencia estratégica en el currículo de manera informada y consciente para los aprendices, susceptible de acometerse en cualquier curso habitual de LE, independientemente de su duración y objetivos.

\section{Referencias bibliográficas}

Bachman, L.F. (1990): Fundamental considerations in Language Testing. Oxford, Oxford University Press.

Bialystok, E. (1990): Communication strategies. A psychological analysis of second-language use. Cambridge, Basis Blackwell.

Brown, A.L. (1978): "Knowing when, where and how to remember: A problem of metacognition". En Glaser, R. (ed.): Advances in instructional psychology, 1. Hillsdale NJ, Erlbaum, págs. 77-165.

Canale, M. y M. Swain (1980): "Theoretical bases of communicative approaches to second language teaching and testing", Applied Linguistics, 1 (1), págs. 1-47.

Canale, M. (1983): "From communicative competence to communicative language pedagogy". En Richards, J. y R. Smith (eds.): Language and Communication. London, Longman, págs. 2-27.

Carver, D. (1984): "Plans, learner strategies and self direction in language learning", System, 12 (2), págs. 123-131.

Chamot, A.U.; Barnhardt, S.; El-Dinary, P.B. y J. Robins (1999): The learning strategies handbook. London, Longman.

Chamot, A.U. y J. Rubin (1994): "Comments on Jenie Rees-Miller's 'A critical appraisal of learner training: Theoretical bases and teaching implications'. Two readers react", TESOL Quarterly, 28 (4), págs. 771-776.

Cohen, A.D. (1990): Language learning insights for learners, teachers and researchers. New York, Newbury House.

Cohen, A.D. (1998): Strategies in learning and using a second language. London, Longman.

Cohen, A.D. (2003): "The learner's side of foreign language learning: Where do styles, strategies, and tasks meet?", IRAL 41, págs. 279-291.

Consejo de Europa (2002): Marco común europeo de referencia para las lenguas: enseñanza, aprendizaje y evaluación. Versión en español, Madrid, Ministerio de Educación, Cultura y Deporte, y Anaya. Disponible en la página web del Instituto Cervantes: http://cvc.cervantes. es/obref/marco. 
Dansereau, D.F. (1985): "Learning strategy research". En Segal, J.W., Chipman, S.F. y R. Glaser (eds.): Thinking and learning skills, 1: Relating Instruction to Research. New Jersey, Lawrence Erlbaum Associates Publishers.

Derry, S.J. y D.A. Murphy (1986): "Designing systems that train learning ability: from theory to practice", Review of Educational Research, 56 (1), págs. 1-39.

Dörnyei, Z. (1995): "On the teachability of communication strategies", TESOL Quarterly, 29 (1), págs. 55-85.

Ellis, G. y B. Sinclair (1989a): Learning to learn English: a course in learner training. Learner's book. Cambridge, Cambridge University Press.

Ellis, G. y B. Sinclair (1989b): Learning to learn English: a course in learner training. Teacher's book. Cambridge, Cambridge University Press.

Ellis, R. (1997): The siudy of second language acquisition. Oxford, Oxford University Press.

Faerch, C., Haastrup, K. y R. Phillipson (1984): Learner language and language learning. Copenhage, Nordisk Forlag.

Faerch, C. y G. Kasper (1984a): "Pragmatic Knowledge: Rules und procedures", Applied Linguistics, 5 (3), págs. 214-278.

Faerch, C. y G. Kasper (1984b): "Two ways of defining communication strategies", Language Learning, 34 (1), págs. 45-64.

Fernández López, S. (2004): "Los contenidos estratégicos". En Sánchez Lobato, J. e I. Santos Gargallo (eds.): Vademécum para la formación de profesores. Enseñar Español como L2/LE. Madrid, SGEL, págs. 853-876.

Flavell, J.H. (1979): "Metacognition and cognitive monitoring: A new area of psychological inquiry", American Psychologist, 34, págs. 906-911.

Gagné, R.M. (1977): The conditions of learning. New York, Holt, Rinehart and Winston (tercera edición).

Graham, S. (1997): Effective language learning. Positive strategies for advanced level language learning. Clevedon, Multilingual Matters.

Grenfell, M. (2002): "Developing learning strategies. Strategy use in the teaching and learning of modern foreign languages". En Swarbrick, A. (ed.): Teaching Modern Foreign Languages in Secondary Schools. A reader. London, Routledge/Falmer, págs. 173-186.

Grenfell, M. y V. Harris (1999): Modern languages and learning strategies. In theory and practice. London, Routledge.

Harris, V. (1997): Teaching learners how to learn. Strategy training in the modern language classroom. London, CILT.

Harris, V. (2002): "Treading a tightrope. Supporting boys to achieve in modern foreign language". En Swarbrick, A. (ed.), Teaching Modern Foreign Languages in Secondary Schools. A reader. London, Routledge/Falmer, págs. 187-202.

Harris, V. y A. Gaspar (2001): Helping learners learn: exploring strategy instruction in language classrooms across Europe. Strasbourg, Council of Europe Publishing.

Hill, S.J. (2004): "La falta de un consenso en la definición de una estrategia de aprendizaje". En Díez, M., Fernández, R. y A. Halbach (eds.): Debate en torno a las estrategias de aprendizaje. Frankfurt am Main, Peter Lang, págs. 21-26.

Holec, H.; Little, D. y R. Richterich (1996): Strategies in language learning and use. Strasbourg, Council of Europe Publishing.

Hosenfeld, C., Arnold, V., Kirchofer, J.L. y L. Wilson (1981): "Second language reading: a curricular sequence for teaching reading strategies", Foreign Language Annals, 14 (5), págs. 415-422.

Instituto Cervantes (1994): La enseñanza del español como lengua extranjera. Plan Curricular del Instituto Cervantes. Madrid, Instituto Cervantes.

Kellerman, E. (1991): "Compensatory strategies in second language research: a critique, a revision, and some (non-) implications for the classroom". En Phillipson, R., Kellerman, E., Selinker, 
L., Sharwood-Smith, M. y M. Swain (eds.): Foreign and second language pedagogy research. Clevedon, Multilingual Matters, págs. 142-161.

Knapp-Potthoff, A. (1982): Fremdsprachenlernen und-lehren: ein Einführung in die Didaktik der Fremdsprachen vom Standpunkt der Fremdsprachenerwerbsforschung. Mainz, Kohlhammer.

Larsen-Freeman, D. y M. Long (1991): Introducción al estudio de la adquisición de segundas lenguas.

Madrid, Gredos. (Versión española, 1994).

Little, D. (1996): "Strategic competence considered in relation to strategic control of the language". En

Holec, H.; Little, D. y R. Richterich: Strategies in language learning and use. Strasbourg, Council of Europe Publishing, págs. 9-37.

Macaro, E. (2001): Learning strategies in foreign and second language classrooms. London, Continuum.

Mayer, R.E. (1988): "Learning strategies: an overwiew". En Weinstein, C. E., Goetz, E. T. y P. Alexander (eds.): Learning and study strategies. New York, Academic Press, págs. 11-22.

Mazzotta, P. (1996): Aprendiendo a aprender. Estrategias de aprendizaje de segundas lenguas y autonomía del estudiante. Sevilla, Mergablum. (Versión española, 2004).

McCombs, B.L. (1988): "Motivational skills training: combining metacognitive, cognitive and affective learning strategies". En Weinstein, C. E., Goetz, E. T. y P. Alexander (eds.): Learning and study strategies. New York, Academic Press, págs. 141-170.

McDonough, S.H. (1995): Strategy and skill in learning a foreign language. London, Edward Arnold.

McDonough, S.H. (1999): "Learner strategies. State of the art article", Language Teaching, 32, págs. 1-18.

Nunan, D. (1989): Designing Tasks for the Communicative Classroom. Cambridge, Cambridge University Press.

O’Malley, J.M. y A.U. Chamot (1990): Learning strategies in second language acquisition. Cambridge, Cambridge University Press.

Oxford, R.L. (1986): Second language learning strategies: current research and implications for practice. Los Angeles, University of California.

Oxford, R.L. (1990): Language learning strategies: what every teacher should know. Boston, Heinle\&Heinle Publishers.

Oxford, R. L. (2004): "Effect of the presence and difficulty of task on strategy use: an exploratory study", International Review of Applied Linguistics in Language Teaching (IRAL), 42 (1), págs. 1-47.

Palmer, D.G. y E.T. Goetz (1988): "Selection and use of study strategies: The role of the studier's beliefs about self and the strategies". En Weinstein, C. E., Goetz, E. T. y P. Alexander (eds.): Learning and study strategies. New York, Academic Press, págs. 41-62.

Peterson, P.L. y S.R. Swing (1983): "Problems in classroom implementation of cognitive strategy instruction". En Pressley, M. y J.R. Levin (eds.): Cognitive Strategy Research. Educational Applications. New York, Springer Verlag.

Rampillon, U. (1985): Lerntechniken im Fremdsprachenunterricht. Ismaning, Hueber.

Rees-Miller, J. (1993): "A critical appraisal of learner training: theoretical bases and teaching implications", TESOL Quarterly, 27 (4), págs. 679-689.

Skehan, P. (1989): Individual differences in second language learning. London, Edward Ardnold.

Sternberg, R.J. (1983): "Criteria for intellectual skills training", Educational Researcher, 12, págs. 6-12.

Sternberg, R.J. (1985): Beyond IQ: A triarchic theory of human intelligence. Cambridge, Cambridge University Press.

Tönshoff, W. (1992): Kognitivierende Verfahren im Fremdsprachenunterricht: Formen und Funktion. Hamburg, Verlag Dr. Kovac. 
Valiente Jiménez, M.J. (2004): "La conciencia del uso de estrategias de comprensión oral en L2". En Díez, M., Fernández, R. y A. Halbach (eds.): Debate en torno a las estrategias de aprendizaje. Frankfurt am Main, Peter Lang, págs. 111-124.

Villanueva, M.L. (1997): "Estilos cognitivos y estilos de aprendizaje. Autonomía y aprendizaje de lenguas". En Villanueva, M. L. e I. Navarro (eds.): Los estilos de aprendizaje de lenguas. Castellón de la Plana, Publicaciones de la Universidad Jaume I, págs. 49-84.

Wenden, A. (1986): "Incorporating learner training in the classroom", System, 14 (3), págs. 315-325. Wenden, A. (1987): "Conceptual Background and Utility". En Wenden, A. y J. Rubin (eds.): Learner Strategies in Language Learning. New Jersey, Englewood Cliffs.

Wenden, A. (1991): Learner strategies for learner autonomy. Cambridge, Prentice Hall.

Wenstein, C.E.; Goetz, E.T. y P. Alexander (eds.) (1988): Learning and study strategies. New York, Academic Press.

Wolff, D. (1998): "Lernstrategien: Ein Weg zu mehr Lernerautonomie", http://paedpsych.jk.uni-linz. ac.at:4711/LEHRTEXTE/Wolff98.html (14-04-2005). 